\title{
Flexible parafoveal encoding of character order supports word predictability effects in Chinese reading: Evidence from eye movements
}

\author{
Min Chang ${ }^{1,2} \cdot$ Lisha Hao ${ }^{1,2} \cdot$ Sainan Zhao ${ }^{1,2} \cdot{\operatorname{Lin~} \mathrm{Li}^{1,2} \cdot \text { Kevin B. Paterson }}^{3} \cdot$ Jingxin Wang ${ }^{1,2}$ \\ Published online: 13 May 2020 \\ (C) The Psychonomic Society, Inc. 2020
}

\begin{abstract}
Several eye-movement studies have revealed flexibility in the parafoveal processing of character-order information in Chinese reading. In particular, studies show that processing a two-character word in a sentence benefits more from parafoveal preview of a nonword created by transposing rather than replacing its two characters. One issue that has not been investigated is whether the contextual predictability of the target word influences this processing of character order information. However, such a finding would provide novel evidence for an early influence of context on lexical processing in Chinese reading. Accordingly, we investigated this issue in an eye-movement experiment using the boundary paradigm and sentences containing two-character target words with high or low contextual predictability. Prior to the reader's gaze crossing an invisible boundary, each target word was shown normally (i.e. a valid preview) or with its two characters either transposed or replaced by unrelated characters to create invalid nonword previews. These invalid previews reverted to the target word once the reader's gaze crossed the invisible boundary. The results showed larger preview benefits (i.e. a decrease in fixation times) for target words following transposedcharacter than substituted-character previews, revealing a transposed-character effect similar to that in previous research. In addition, a word predictability effect (shorter fixation times for words with high than low predictability) was observed following both valid and transposed-character previews, but not substituted-character previews. The findings therefore reveal that context can influence an early stage of lexical processing in Chinese reading during which character order is processed flexibly.
\end{abstract}

Keywords Eye movements $\cdot$ Word predictability $\cdot$ Parafoveal preview $\cdot$ Transposed-character effect $\cdot$ Chinese reading

\section{Introduction}

Substantial evidence from research with alphabetic scripts indicates flexibility in the spatial encoding of orthographic information (Grainger, Dufau, \& Ziegler, 2016). Much of this research comes from studies assessing the influence of brief, masked presentations of transposed-letter primes on lexical decisions (i.e. word/nonword judgements) for subsequent

Kevin B. Paterson

kbp3@le.ac.uk

Jingxin Wang

wjxpsy@126.com

1 Academy of Psychology and Behaviour, Tianjin Normal University, Hexi District, Tianjin 300374, China

2 Faculty of Psychology, Tianjin Normal University, Tianjin, China

3 Department of Neuroscience, Psychology and Behaviour, University of Leicester, University Road, Leicester LE1 9HN, UK target words (e.g. Perea \& Lupker, 2003). These show that, for a target word like 'judge', primes containing letter transpositions (e.g. 'jugde') produce responses that are faster than following primes with letter substitutions (e.g. 'jupbe'), and often not much slower than responses following correctly spelled primes (e.g. 'judge'). As masked primes are assumed to influence an early stage of word recognition (Forster, Mohan, \& Hector, 2003), the findings suggest that letter position, but not letter identity, is processed flexibly early in word recognition. Moreover, such findings have been central to the development of models of visual word recognition (see Norris, 2013).

Other research shows that transposing letters in words is associated with longer reading times for text (Rayner et al., 2006; White et al., 2008). However, eye-movement studies using the boundary paradigm show transposed-letter effects during parafoveal processing similar to those in masking priming (e.g. Johnson, Perea, \& Rayner, 2007). In this paradigm, an invisible boundary is placed in front of a target word in a sentence. Prior to the reader's gaze crossing the boundary, 
the target word is shown normally (i.e. a valid preview, e.g. 'clerk'), or with letters transposed (e.g. 'clrek') or replaced by other letters (e.g. 'cohrk') to create an invalid preview. Once the reader's gaze crosses the boundary, the display reverts to the target word, within the time it takes the reader to make an eye movement, so that readers are unaware a change has been made. In this way, preview effects on subsequent target word processing can be examined. Studies using this paradigm show larger benefits for transposed-compared to substituted-letter previews, suggesting letter-position information is processed flexibly during parafoveal processing. This research has primarily been conducted in alphabetic scripts like English. However, recent studies have examined whether similar effects are observed in Chinese reading. Chinese is created from box-like logograms called characters, with most words comprising two (or more) characters (Hoosain, 1992), although word boundaries are not visually demarcated in text (Li et al., 2015). Studies have therefore investigated whether a word's character order is processed strictly in Chinese reading.

An early study by Taft, Zhu and Peng (1999) used a paradigm in which participants made lexical decisions for twocharacter words (and nonwords) that could form a word, or not, when their characters were transposed. Lexical decisions were slower for transposable words and nonwords relative to non-transposable controls, which Taft et al. took as evidence for flexible character-order processing. They argued that presentation of a transposable word activates lexical entries for both the correct word and its transposed-character counterpart (i.e. 领带 activates both 领带, meaning 'tie', and 带领, meaning 'lead'), with competition between these slowing lexical decisions. Similarly, they argued that, as a transposable nonword presentation can activate the lexical entry for its base word, this will interfere with the decision to reject the nonword presentation, slowing lexical decisions for transposable nonwords relative to non-transposable controls.

Other evidence from studies using conventional masked priming methods (Gu, Li, \& Liversedge, 2015: Experiment 1) supports this view, by showing faster responses to target words (e.g. 吝啬) following a transposed-character nonword prime (e.g. 啚 吝) than unrelated nonword prime (e.g. 菠录). The explanation here is that the brief, masked presentation of a transposable nonword activates the lexical entry for its base word, facilitating the recognition of that word when subsequently it is presented as a target. Similarly, eye-movement studies using the boundary paradigm show that target word processing benefits more from parafoveal preview of a transposed- than substituted-character nonword (Gu et al., 2015: Experiment 2; Gu \& Li, 2015). In this paradigm, an invisible boundary is placed immediately prior to a target word and, before the reader's gaze crosses this boundary, the target word is displayed normally (i.e. a valid preview) or replaced by a nonword created by either transposing or replacing its two characters (invalid transposed-character or substituted- character previews). As soon as the reader's gaze crosses the boundary, the invalid displays revert to the target word and the experimenter can examine the effects of the different previews on the subsequent processing of the target word. Finding that a transposed-character preview facilitates processing relative to a substituted-character preview suggests that preview of a transposed-character nonword can activate the lexical entry for its base word, helping lexical identification of that word following the display change. Yang (2013) additionally showed that transposed-character previews that formed words with either a similar or a different meaning to the target produced similar benefits relative to a control preview, indicating that the effect is due to flexible character order encoding rather than semanticrelatedness. Studies to date therefore provide good evidence for flexible parafoveal processing of character order in Chinese reading.

An outstanding question concerns whether contextual expectations can influence parafoveal processing of characterorder information during Chinese reading. Such effects are theoretically important, as they may provide novel evidence that context influences an early stage of word identification associated with parafoveal processing of linguistic information. Numerous boundary paradigm studies in alphabetic languages show that predictability effects when previews are identical to the target word (e.g. cake) are reduced for orthographically similar, substituted-letter previews (e.g. cahc), and eliminated for orthographically dissimilar previews (e.g. pies; Balota, Pollatsek, \& Rayner, 1985; see Staub, 2015), with similar effects for Chinese (Chang et al., 2020). These findings suggest that, in high-predictability contexts, readers assess the fit of parafoveal information with their expectations. As parafoveal processing is degraded due to limitations in retinal acuity (Hilz \& Cavonius, 1974), coarse perceptual information that fits those expectations (i.e. a visually similar preview) may facilitate subsequent processing of target word information, wherease parafoveal information that does not fit with predictions (i.e. a visually dissimilar preview) will not. An important question, therefore, is whether word predictability effects also are observed for transposed- relative to substituted-character previews, as this would reveal that contextual predictability and flexible character order processing can conspire to influence parafoveal processing. One possibility is that predictability might facilitate the processing of transposed-character relative to substituted-character previews. However, a study by Luke and Christianson (2012), using a novel paradigm combining self-paced word-by-word alphabetic reading and masked priming, found an advantage for transposed-letter over substituted-letter primes in nonconstraining contexts was eliminated when the target word was strongly predicted. This was taken to show that contextual prediction can be highly lexically specific, including predicting the specific identity and order of letters in words (Laszlo \& Federmeier, 2009). However, this requires further 
investigation as the study used a non-standard method and manipulated context across experiments (low- and highconstraint materials were used in different experiments).

Accordingly, the present experiment assessed the influence of word predictability on the transposed-character effect in Chinese reading. Participants read sentences where an invisible boundary was placed immediately before a two-character target word that was either highly predictable or less predictable from the prior sentence context (Table 1). Before the reader's gaze crossed this boundary, the target was shown normally, as a valid preview, or with its characters either transposed or substituted by orthographically dissimilar characters to create invalid nonword previews, following the same procedure as previous research (Gu et al., 2015). We expected highly predictable words to yield shorter reading times than less predictable words, in line with research on word predictability effects in Chinese reading (e.g. Rayner et al., 2005; Zhao et al., 2019). We also expected to replicate a larger preview benefit for transposed- relative to substitutedcharacter nonwords, consistent with flexible parafoveal processing of character order in previous research (Gu et al., 2015). The crucial question was whether predictability would modulate this effect. Following Luke and Christianson (2012), we might expect a transposed-character effect for less predictable words to be reduced or eliminated for highly predictable words. Alternatively, if lexical prediction is supported by flexible parafoveal encoding of character order, predictability effects may be larger following transposed- than substituted-character previews.

\section{Method}

Eethics statement The study was approved by the research ethics committee in the Academy of Psychology and
Behavior at Tianjin Normal University and conducted in accordance with the principles of the Declaration of Helsinki.

Participants Sixty young adults aged 18-24 years $(M=20)$ from Tianjin Normal University participated. An additional three participants were replaced as they noticed a large proportion of display changes. All were native Mandarin readers, screened for normal acuity (greater than 20/40 in Snellen values) using a Tumbling E eye chart (Taylor, 1978), and naïve to the purpose of the experiment. A power analysis based on the gaze-duration effects in Experiment 2 of Gu et al. (2015), using the SIMR package (Green \& McLeod, 2016) in R (R Development Core Team, 2016), suggested the experiment was appropriately powered to detect a transposed-character effect (power $>80 \%$ ). Note that previous research has not reported effect sizes for word-skipping, precluding power analysis based on this measure.

Stimuli and design Stimuli were 90 Chinese sentence frames, each containing a two-character target word. Following procedures similar to those used in other studies of predictability effects in alphabetic languages and Chinese (Rayner et al., 2005; Rayner \& Well, 1996; Zhao et al., 2019), words were selected to have high or low predictability from the prior sentence context using a cloze procedure with sentences truncated immediately before the target word. Fifteen young adults who did not take part in the experiment provided the next word in the sentence. A word was considered highly predictable if more than $75 \%$ of participants guessed it to be the next word, and as less predictable if fewer than $20 \%$ guessed it to be the next word. See Table 2 for selected words characteristics. High- and low-predictability words were matched for word frequency and first- and second-character frequency using the SUBTLEX-CH database (Cai \& Brysbaert, 2010), and

Table 1 An example stimulus. Preview stimuli and target words are underlined and vertical lines indicate the invisible boundary

\begin{tabular}{|c|c|c|}
\hline Predictability & Preview & Stimulus Example \\
\hline \multirow[t]{3}{*}{ High } & Identity & $\begin{array}{l}\text { 她脖子上戴的那串项链项链奶奶留给她的遗 } \\
\text { 物。 }\end{array}$ \\
\hline & Transposed & $\begin{array}{l}\text { 她脖子上戴的那串链项|项链奶奶留给她的遗 } \\
\text { 物。 }\end{array}$ \\
\hline & Substituted & $\begin{array}{l}\text { 她脖子上戴的那串湖姑|项链奶奶留给她的遗 } \\
\text { 物。 }\end{array}$ \\
\hline \multirow[t]{3}{*}{ Low } & Identity & $\begin{array}{l}\text { 她脖子上戴的那串铃铛|铃铛 奶奶留给她的遗 } \\
\text { 物。 }\end{array}$ \\
\hline & Transposed & $\begin{array}{l}\text { 她脖子上戴的那串铛铃铃铛奶奶留给她的遗 } \\
\text { 物。 }\end{array}$ \\
\hline & Substituted & $\begin{array}{l}\text { 她脖子上戴的那串账伟|铃铛 奶奶留给她的遗 } \\
\text { 物。 }\end{array}$ \\
\hline
\end{tabular}

The high-predictable target word 项链 means 'necklace' and the low-predictable target word 铃铛 means 'bell'. The sentence translates as 'The string of necklaces/bells worn on her neck is the relic left by her grandmother' 
Table 2 Mean target word properties

\begin{tabular}{|c|c|c|c|c|}
\hline Word property & High & Low & $t$ & $p$ \\
\hline Predictability $(\%)$ & $89(9)$ & $1(3)$ & 82.61 & $<.001$ \\
\hline Word frequency (counts/million) & $86(125)$ & $58(145)$ & 1.47 & .15 \\
\hline First character frequency (counts/million) & $660(768)$ & $582(870)$ & .69 & .49 \\
\hline Second character frequency (counts/million) & $663(1,035)$ & $823(1,453)$ & .90 & .37 \\
\hline Visual complexity & $17.3(4.18)$ & $16.8(4.19)$ & 1.00 & .32 \\
\hline Naturalness & $6.43(.29)$ & $6.33(.50)$ & 1.80 & .08 \\
\hline
\end{tabular}

The standard deviation of the mean is shown in parentheses

for visual complexity in terms of number of character strokes. Ten participants evaluated sentence naturalness (using a 7point scale, ranging from $1=$ entirely unnatural to $7=$ entirely natural). Ratings were high with no significant difference between sentences with high- and low-predictability target words. Sentences averaged 22 characters long (range $=18$ 31 characters) and target words always appeared near their center.

Sentences were presented using the boundary paradigm (Rayner, 1975), with an invisible boundary placed immediately before the target word. Valid previews were the high- and low- predictability words. Following the same procedure as previous research (Gu et al., 2015), tranposed-character and substituted-character nonword previews were created by either transposing a word's characters or replacing both characters with orthographically dissimilar characters. Characters in substituted-character previews could not form a word with characters to the left or right and were matched with the target words for first- and second-character frequency $(t \mathrm{~s}<1.4, p \mathrm{~s}>$ $.18)$, and number of strokes $(t(89)=1.00, p=.32)$.

The experiment used a within-subjects design with the factors word predictability (high, low) preview type (valid, invalid transposed-character, invalid substituted-character) as independent variables and eye-movement measures as dependent variables. We adopted a counterbalanced design in which each participant read one version of each sentence frame and equal numbers of sentences in each condition.

Apparatus and procedure An SR Eyelink 1000 plus eye tracker tracked right-eye movements during binocular viewing at 1,000 Hz. Stimuli were displayed in Song 34-point font as black-on-white text on a high-resolution $(1,920 \times 1,080$ pixels) monitor with a fast refresh rate $(144 \mathrm{~Hz})$. At $58-\mathrm{cm}$ viewing distance, each character subtended $1.2^{\circ}$ and so was of normal size for reading.

Participants took part individually and were instructed to read normally and for comprehension. At the start of the experiment, a three-point horizontal calibration procedure was performed across the same line as each sentence presentation (ensuring $.30^{\circ}$ or better spatial accuracy for all participants). Calibration accuracy was checked before each trial and the eye tracker recalibrated as required to maintain high spatial accuracy. At the start of each trial, a fixation square equal in size to one character was presented on the left side of the screen. Once the participant fixated this location, a sentence was presented with the first character replacing the square. Participants pressed a response key once they finished reading each sentence. This was replaced on $30 \%$ of trials by a comprehension question requiring a yes/no button-press response. At the end of the experiment, participants were asked if they noticed display changes. Three reported seeing more than $10 \%$. Data for these participants were excluded from analyses and the participants replaced. All other participants reported seeing at most $5 \%$ display changes. The experiment lasted approximately $30 \mathrm{~min}$ for each participant.

\section{Results}

Accuracy answering comprehension questions was high for all participants $(>90 \%)$. Following standard procedures, short $(<80 \mathrm{~ms})$ and long $(>1,200 \mathrm{~ms})$ fixations were removed (affecting $4.4 \%$ of fixations). Trials with track-loss or error were also excluded (affecting $21(<1 \%)$ trials), as were trials for sentences receiving fewer than than six fixations (affecting 355 (6.6\%) trials; following Zhang et al., 2019, although the same pattern of effects was observed when this exclusion criterion was not applied), trials in which an eye-blink was made within the boundary region or when fixating the target word (affecting 94 (1.7\%) trials), and trials where display changes were delayed (i.e. implemented $>12 \mathrm{~ms}$ into the next fixation, affecting 656 (12\%) fixations). We also excluded trials in which a saccade triggered a display change but terminated to the left of the larget word (i.e. a ' $\mathrm{j}$-hook', affecting $145(2.7 \%)$ trials). In total, 1,271 (23.5\%) trials were removed. The remaining data were analysed by linear mixed-effects models (LMEs, Baayen, Davidson, \& Bates, 2008) for continuous variables and generalized mixed-effects models for binomial variables, using the lme4 package (Version 1.1-21; Bates, Mächler, Bolker, \& Walker, 2015) in R (R Development Core Team, 2016). For all measures, models with maximum 
random-effects structure were used (Barr, Levy, Scheepers, \& Tily, 2013), with predictability, preview and their interaction as fixed factors, and participants and stimuli as crossed random effects. If models did not converge, the random-effects structure was reduced by first trimming this for stimuli, starting with removal of random-effect correlations, then random slopes. Contrasts of main effects and contrasts to examine interactions were defined using sliding contrasts (the contr.sdif function) in the MASS package (Version 7.3-47; Venables \& Ripley, 2002). Log-transformed fixation-time effects are reported alongside untransformed means. Following convention, $t / z$ values $>1.96$ were considered significant.

We report word-level analyses for the target words, including measures informative about first-pass reading, i.e. the initial processing of a target word prior to a fixation to its right or a regression. These comprised word-skipping (SKIP, probability of not fixating a word during first-pass reading), firstfixation duration (FFD, duration of the first fixation on a word during first-pass reading), single-fixation duration (SFD, duration of the first fixation on a word receiving only one firstpass fixation), gaze duration (GD, sum of all first-pass fixations on a word) and regressions-out (RO, probability of a first-pass regression from a word). We report total reading time (TRT, sum of all fixations on a word) and regressionsin (RI, probability of a regression back to a word) as measures of later processing. These analyses were based on $2,438 \mathrm{ob}-$ servations for FFD, 2,254 observations for SFD (wth $42 \%$ of target words receiving a single first-pass fixation), 2,438 observations for GD, and 3,074 observations for TRT.

Target word means are shown in Table 3 and statistical effects summarized in Table 4. Predictability effects, marginal in FFD and significant in GD and TRT, were due to longer reading times for low- than high-predictability words. Marginal effects in regressions suggested readers made more regressions to the less predictable words. Preview effects were significant in target-word reading times (FFD, SFD, GD, TRT; see Fig. 1). Sliding contrasts showed these were longer for substituted-character compared to transposed-character previews, and for transposed-character compared to valid previews, revealing a transposed-character effect similar to previous research (Gu et al., 2015). A preview effect in wordskipping was due to reduced skipping following substitutedcharacter compared to transposed-character previews, with no difference between transposed-character and valid previews, indicating less skipping when the preview was uninformative about target-word identity. Finally, readers made more regressions back to target words following transposed-character compared to valid previews, with no difference between transposed-character and substituted-character previews, indicating that invalid previews caused readers to re-inspect words more often.

Interactions between word predictability and the contrast between transposed-character and substitutedcharacter previews in FFD and SFD were due to significant word predictability effects in these early fixation-time measures following transposed-character but not substituted-character previews (FFD, transposed-character preview $=12-\mathrm{ms}$ effect, substituted-character preview $=$ $-13-\mathrm{ms}$ effect; SFD, transposed-character preview $=12$ ms effect, substituted-character preview $=-13-\mathrm{ms}$ effect; see Fig. 1). The same pattern of effect was observed in GD, although not significantly. A similar effect in wordskipping was due to lower skipping following substituted-character than transposed-character previews in high- than low-predictability contexts, most likely because substituted-character previews mismatched with contextual expectations in the high predictability condition. By comparison with these effects, no interactions between word predictability and the contrast between the transposed-character previews and valid previews in FFD and SFD were observed (FFD, valid preview $=10$-ms effect; SFD, valid preview $=9-\mathrm{ms}$ effect). The findings therefore indicate that transposed-character previews, but not substituted-character previews, supported word

Table 3 Means for target word measures

\begin{tabular}{|c|c|c|c|c|c|c|}
\hline \multirow[t]{2}{*}{ Measure } & \multicolumn{3}{|c|}{ High predictability } & \multicolumn{3}{|c|}{ Low predictability } \\
\hline & Identical & Transposed & Substituted & Identical & Transposed & Substituted \\
\hline $\operatorname{SKIP}(\%)$ & $44(2)$ & $42(2)$ & $34(2)$ & $43(2)$ & $41(2)$ & $41(2)$ \\
\hline $\mathrm{FFD}(\mathrm{ms})$ & $222(3)$ & $248(5)$ & $289(5)$ & $232(4)$ & $260(5)$ & $276(5)$ \\
\hline $\mathrm{SFD}(\mathrm{ms})$ & $222(3)$ & $246(5)$ & $289(6)$ & $231(4)$ & $258(5)$ & $276(6)$ \\
\hline GD (ms) & $227(4)$ & $262(6)$ & $311(6)$ & $242(5)$ & $279(6)$ & $313(7)$ \\
\hline RO (\%) & $10(2)$ & $9(1)$ & $17(2)$ & $11(2)$ & $12(2)$ & $19(2)$ \\
\hline TRT (ms) & $278(8)$ & $331(11)$ & $371(11)$ & $289(8)$ & $337(9)$ & $377(10)$ \\
\hline $\mathrm{RI}(\%)$ & $11(2)$ & $18(2)$ & $15(2)$ & $14(2)$ & $16(2)$ & $20(2)$ \\
\hline
\end{tabular}

Note. All measures are in milliseconds except word-skipping (SKIP), regressions-out (RO) and regressions-in (RI), which are percentage probabilities. The standard error of the mean is shown in parentheses 
Table 4 Summary of statistical effects (continuous variables were log-transformed)

\begin{tabular}{|c|c|c|c|c|c|c|c|c|}
\hline & & SKIP & FFD & SFD & GD & TRT & RO & RI \\
\hline \multirow[t]{4}{*}{ Intercept } & $\mathrm{B}$ & -.40 & 5.47 & 5.47 & 5.51 & 5.64 & -2.22 & -1.82 \\
\hline & CI & {$[-.58,-.23]$} & {$[5.43,5.5]$} & {$[5.43,5.5]$} & {$[5.47,5.55]$} & {$[5.47,5.55]$} & {$[-2.53,-1.95]$} & {$[-2.03,-1.62]$} \\
\hline & $S E$ & .09 & .02 & .02 & .02 & .03 & .14 & .1 \\
\hline & $t / z$ & -4.59 & 311.86 & 306.26 & 279.17 & 222.15 & -15.35 & -17.91 \\
\hline \multirow[t]{4}{*}{ Predictability } & B & .05 & .02 & .02 & .04 & .04 & .16 & .16 \\
\hline & $\mathrm{CI}$ & {$[-.08, .17]$} & {$[-.01, .04]$} & {$[-.01, .04]$} & {$[.01, .07]$} & {$[.01, .07]$} & {$[-.11, .42]$} & {$[-.06, .40]$} \\
\hline & $S E$ & .07 & .01 & .01 & .01 & .02 & .13 & .12 \\
\hline & $t / z$ & .73 & 1.53 & 1.37 & $2.69 *$ & $2.18^{*}$ & 1.2 & 1.42 \\
\hline \multirow{4}{*}{$\begin{array}{l}\text { Preview } \\
\text { (Transposed-Identical) }\end{array}$} & B & -.08 & .1 & .09 & .12 & .14 & -.09 & .39 \\
\hline & CI & {$[-.24, .08]$} & {$[.07, .13]$} & {$[.06, .13]$} & {$[.08, .15]$} & {$[.08, .15]$} & {$[-.44, .26]$} & {$[.1, .69]$} \\
\hline & $S E$ & .08 & .02 & .02 & .02 & .02 & .17 & .15 \\
\hline & $t / z$ & -.98 & $6.17^{*}$ & $5.96^{*}$ & $6.61^{*}$ & $6.27 *$ & -.54 & $2.63 *$ \\
\hline \multirow{4}{*}{$\begin{array}{l}\text { Preview } \\
\text { (Substituted-Transposed) }\end{array}$} & $\mathrm{B}$ & -.21 & .1 & .11 & .14 & .11 & .8 & .07 \\
\hline & $\mathrm{CI}$ & {$[-.37,-.05]$} & {$[.07, .13]$} & {$[.08, .14]$} & {$[.10, .17]$} & {$[.10, .17]$} & {$[.50,1.12]$} & {$[-.19, .33]$} \\
\hline & $S E$ & .08 & .02 & .02 & .02 & .02 & .15 & .13 \\
\hline & $t / z$ & $-2.59^{*}$ & $6.74 *$ & $6.78 *$ & $8.12 *$ & $5.41 *$ & $5.23 *$ & .52 \\
\hline \multirow{4}{*}{$\begin{array}{l}\text { Predictability } \times \text { Preview } \\
\text { (Transposed-Identical) }\end{array}$} & B & 0 & .01 & .01 & .02 & .02 & .32 & -.41 \\
\hline & $\mathrm{CI}$ & {$[-.32, .31]$} & {$[-.05, .07]$} & {$[-.05, .08]$} & {$[-.05, .08]$} & {$[-.05, .08]$} & {$[-.38,1.02]$} & {$[-.10, .18]$} \\
\hline & $S E$ & .16 & .03 & .03 & .04 & .04 & .34 & .3 \\
\hline & $t / z$ & -.02 & .44 & .43 & .45 & .39 & .92 & -1.38 \\
\hline \multirow{4}{*}{$\begin{array}{l}\text { Predictability } \times \text { Preview } \\
(\text { Substituted-Transposed) }\end{array}$} & B & .38 & -.09 & -.08 & -.06 & -.04 & -.11 & .5 \\
\hline & CI & {$[.06, .70]$} & {$[-.15,-.03]$} & {$[-.14,-.02]$} & {$[-.13,0]$} & {$[-.13,0]$} & {$[-.74, .50]$} & {$[-.02,1.03]$} \\
\hline & $S E$ & .16 & .03 & .03 & .03 & .04 & .3 & .27 \\
\hline & $t / z$ & $2.32 *$ & $-2.89 *$ & $-2.51^{*}$ & -1.90 & -.98 & -.38 & 1.89 \\
\hline
\end{tabular}

Note. Asterisks indicate significant effects where $t>1.96$

CI $95 \%$ confidence interval

Syntax for the LME models was: FFD, GD: lmer (depvar Predictability * Preview + $(1 \mid$ pp $)+(1 \mid$ Item), datafile); SFD, TRT: lmer (depvar Predictability * Preview + (1+ Predictability $\mid$ pp $)+(1 \mid$ Item $)$, datafile); SKIP, RI, RO: glmer (depvar $\sim$ Predictability * Preview + (1 $\mid$ pp $)+(1 \mid$ Item $)$, datafile, family=binomial)

predictability effects during early processing of target words. No other interaction effects were significant.

Bayes factors (Kass \& Raftery, 1995) were calculated to assess interaction effects in fixation time measures (note this technique is not applicable to discontinuous variables like word-skipping). These were computed separately for models examining contrasts between identical and transposedcharacter previews, and transposed-character and substituted-character previews. The analyses were conducted using the $1 \mathrm{mBF}$ function within the BayesFactor package (Version 0.9.12-2; Morey, Roudner, \& Jamil, 2015) in R (R Core Team, 2016), assessing support for $\mathrm{H}_{1}$ over $\mathrm{H}_{0}$ (i.e. $\mathrm{BF}_{10}$; see, e.g., Morey \& Rouder, 2011) by comparing models incorporating both main effects and an interaction between preview and predictability against models containing only main effects of these variables. Analyses were conducted with scaling factor for g-priors set to 0.5, using 100,000 Monte Carlo iterations, and participants and items specified as random factors. Following Vandekerckhove, Matzke, and Wagenmakers (2015; derived from Jeffreys, 1961), Bayes factors $(\mathrm{BFs})>3$ were taken to provide weak to moderate support for a model including an interaction effect, and BFs $>10$ to provide strong support for this model, while BFs $<1$ provided evidence against a model including an interaction effect and in favor of a model with only main effects.

FFD and SFD are early measures of target word processing that in LME analyses produced an interaction effect for the transposed-character versus substituted-character contrast but not the valid versus transposed-character contrast. This was consistent with our prediction that transposed-character, but not substituted-character, previews can support predictability effects during early processing. BFs for these measures provided strong evidence for a model without an interaction (i.e. main effects only) for the valid versus transposed-character contrast $\left(\mathrm{FFD}, \mathrm{BF}_{10}=.08 ; \mathrm{SFD}, \mathrm{BF}_{10}=.11\right)$, and weak to moderate evidence for a model with an interaction effect for 

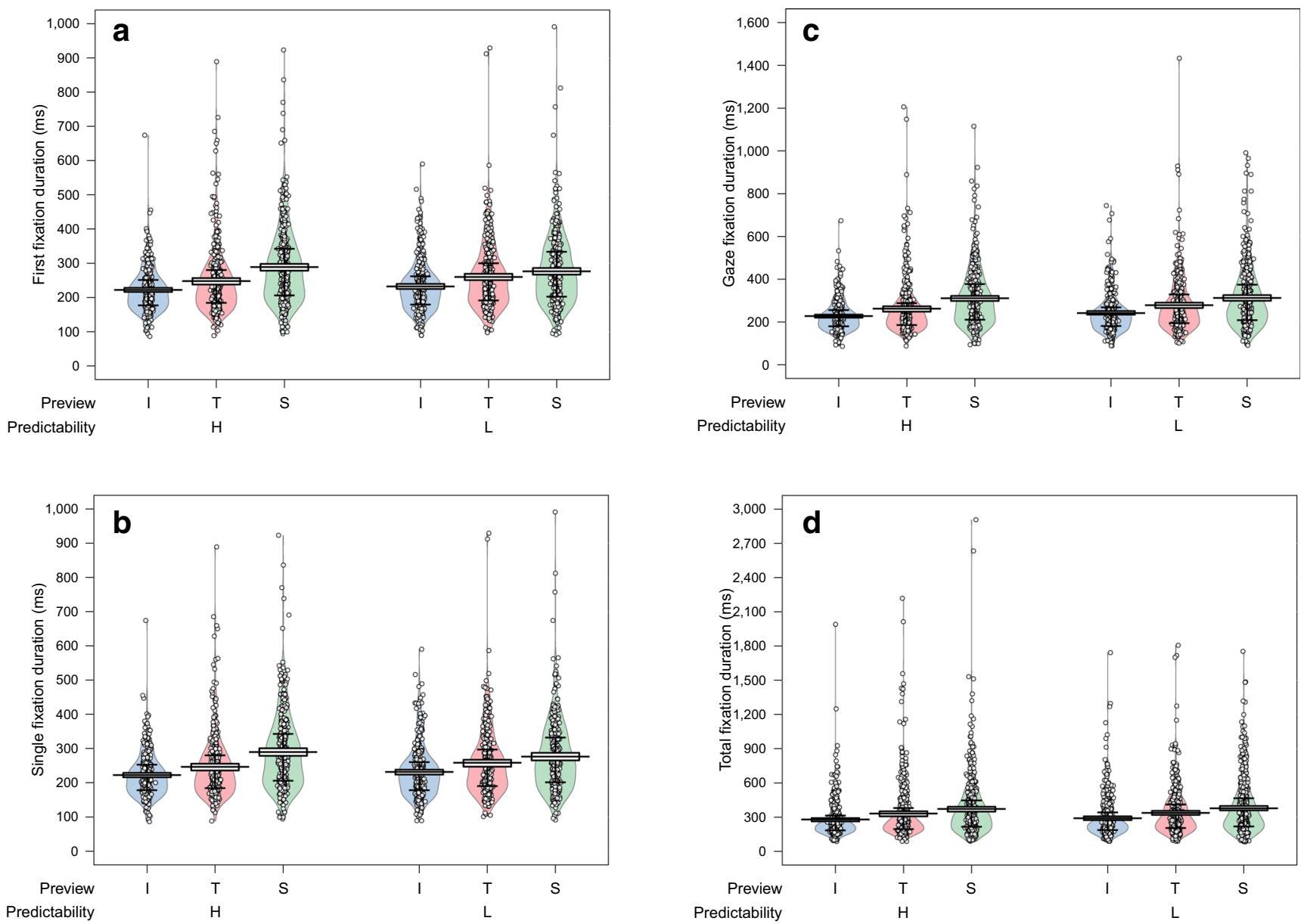

Fig. 1 Pirate plots creating using the Yarrr package in R (Version 0.1.5, Phillips, 2017) depicting effects of preview type on fixation times for high- and low-predictability target in (A) first-fixation durations, (B) single-fixation durations, (C) gaze durations and (D) total reading times

the transposed-character versus substituted-character contrast $\left(\mathrm{FFD}, \mathrm{BF}_{10}=3.64 ; \mathrm{SFD}, \mathrm{BF}_{10}=1.62\right)$. For effects in later measures (GD, TRT), we observed no interaction affects in LME analyses for valid versus transposed-character or transposed-character versus substituted-character contrasts. BFs confirmed these effects by favoring models with main effects and no interaction (all $\mathrm{BF}_{10}<1$ ). Consequently, $\mathrm{BFs}$ provide partial, weak-to-moderate support for interaction effects in early processing measures, and confirmatory evidence for the absence of interaction effects in later processing.

\section{Discussion}

With the present experiment, we used the boundary paradigm to investigate whether transposed-character nonword previews can support word predictability effects in Chinese reading. The results showed a standard word predictability effect in reading times for target words, with shorter reading times (in GD and TRT) for more predictable words, in line with previous Chinese findings (Rayner et al., 2005; Zhao et al., 2019). However, unlike in these previous studies, we did not observe a predictability effect in word-skipping. This may be explained by comparatively high skipping rates in our experiment compared to these previous studies. The presence of effects in reading times nevertheless shows that our manipulation of word predictability was effective.

We also replicated the transposed-character effect reported previously (Gu et al., 2015), by showing that target-word identification was facilitated by transposed-character relative to substituted-character previews. Our findings therefore provide further evidence that character order is encoded flexibly early during Chinese reading. However, as readers made more regressions to invalid than valid previews, it seems a cost for invalid previews is not entirely eliminated by this flexibility in character order processing.

Crucially, we also observed interactions between word predictability and parafoveal preview, such that effects of word predictability during early target word processing (i.e. in FFD and SFD) were obtained for transposed- but not substitutedcharacter previews. This is theoretically important as it provides a novel demonstration that contextual information can guide parafoveal processing of character order, implicating contextual knowledge in this very early aspect of the spatial 
encoding of orthographic information during Chinese reading. This finding contrasts with those reported by Luke and Christianson (2012) in experiments using a novel paradigm combining self-paced alphabetic reading with masked priming. In these experiments, transposed-nonword priming effects in low-constraining contexts were eliminated in highconstraining contexts, which Luke and Christianson attributed to prediction of the specific identity and order of letters in high-constraining contexts conflicting with prime information. Such findings are consistent with the claim that contextual prediction can be highly lexically-specific (e.g. Laszlo \& Federmeier, 2009). One possibility is that this difference in findings represents a cross-script difference in the specificity of contextual prediction, perhaps because context does not strongly predict specific character identity or order in Chinese reading due to the large variety of characters and synonyms in this language (Campbell, 1997). However, as already noted, Luke and Christianson used non-standard methods and compared context effect across experiments. Consequently, further research that investigates contextual influences on the parafoveal processing of spatial information using standard eye-movement methods is required to confirm whether effects differ for alphabetic compared to character-based scripts.

Acknowledgements Min Chang and Lisha Hao are joint first authors and Jingxin Wang and Kevin Paterson are joint corresponding authors. The research was supported by a grant from the National Science Foundation of China to Jingxin Wang (81771823) and a 1000 Talents Visiting Professorship to Kevin Paterson. Lisha Hao designed the experiment with help from the other authors. Lisha Hao, Sainan and Lin Li designed the stimuli, Lisha Hao collected the data. Lisha Hao and Min Chang analyzed the data. Lisha Hao, Min Chang and Kevin Paterson wrote the manuscript. We thank Xue Zhang for helping with the data collection. The authors have no conflicts of interest.

Open Practices Statement Data files and related resources are available from the University of Leicester online Figshare repository: https://doi. org/10.25392/leicester.data.12122952.v1. The experiment was not preregistered.

\section{References}

Baayen, R. H., Davidson, D. J., \& Bates, D. M. (2008). Mixed-effects modeling with crossed random effects for subjects and items. Journal of Memory and Language, 59, 390-412. doi:https://doi. org/10.1016/j.jml.2007.12.005

Balota, D. A., Pollatsek, A., \& Rayner, K. (1985). The interaction of contextual constraints and parafoveal visual information in reading. Cognitive Psychology, 17, 364-390. doi:https://doi.org/10.1016/ 0010-0285(85)90013-1

Barr, D. J., Levy, R., Scheepers, C., \& Tily, H. J. (2013). Random effects structure for confirmatory hypothesis testing: Keep it maximal. Journal of Memory and Language, 68, 1-43. doi:https://doi.org/10. 1016/j.jml.2012.11.001

Bates, D., Mächler, M., Bolker, B. M., \& Walker, S. C. (2015). Fitting linear mixed-effects models using lme4. Journal of Statistical Software, 67, 1-48. doi:https://doi.org/10.18637/jss.v067.i01
Cai, Q., \& Brysbaert, M. (2010). SUBTLEX-CH: Chinese Word and Character Frequencies Based on Film Subtitles. PLOS ONE, 5, e10729. doi:https://doi.org/10.1371/journal.pone.0010729

Campbell, G. L. (1997). Handbook of scripts and alphabets. Psychology Press.

Chang, M., Zhang, K., Hao, L., Zhao, S., McGowan, V. A., Warrington, K. L., Paterson, K. B., Wang, J., \& Gunn, S. C. (2020). Word predictability depends on parafoveal preview validity in Chinese reading. Visual Cognition, 28, 33-40. https://doi.org/10.1080/ 13506285.2020.1714825

Forster, K. I., Mohan, K., \& Hector, J. (2003). The mechanics of masked priming. In S. Kinoshita and S. J. Lupker (Eds.), Masked Priming: The State of the Art (pp. 2-20). Psychology Press; Hove, UK. https:// doi.org/10.4324/9780203502846

Grainger, J., Dufau, S., \& Ziegler, J. C. (2016). A vision of reading. Trends in Cognitive Sciences, 20, 171-179. https://doi.org/10. 1016/j.tics.2015.12.008

Green, P. and MacLeod, C. J. (2016). SIMR: An R package for power analysis of generalized linear mixed models by simulation. Methods in Ecology and Evolution, 7, 493-498. doi: https://doi.org/10.1111/ 2041-210X.12504

Gu, J., \& Li, X. (2015). The effects of character transposition within and across words in Chinese reading. Attention, Perception, \& Psychophysics, 77, 272-281. doi: https://doi.org/10.3758/s13414014-0749-5

Gu, J., Li, X., \& Liversedge, S. P. (2015). Character order processing in Chinese reading. Journal of Experimental Psychology: Human Perception and Performance, 41, 127-137. https://doi.org/10. 1037/a0038639

Hilz, R., \& Cavonius, C. R. (1974). Functional organization of the peripheral retina: Sensitivity to periodic stimuli. Vision Research, 14, 1333-1337.

Hoosain, R. (1992). Psychological reality of the word in Chinese. In H.C. Chen \& O. J. L. Tzeng (Eds.), Advances in Psychology, 90. Language Processing in Chinese (pp. 111-130). Oxford, England: North-Holland. doi:https://doi.org/10.1016/S0166-4115(08)618890

Jeffreys, H. (1961). Theory of probability (3rd). Oxford, UK: Oxford University Press.

Johnson, R. L., Perea, M., \& Rayner, K. (2007). Transposed-letter effects in reading: Evidence from eye movements and parafoveal preview. Journal of Experimental Psychology: Human Perception and Performance, 33, 209-229. https://doi.org/10.1037/0096-1523.33. 1.209

Kass, R. E., \& Raftery, A. E. (1995). Bayes factors. Journal of the American Statistical Aassociation, 90, 773-795.

Laszlo, S., \& Federmeier, K. D. (2009). A beautiful day in the neighborhood: An event-related potential study of lexical relationships and prediction in context. Journal of Memory and Language, 61, 326338. doi: https://doi.org/10.1016/j.jml.2009.06.004

Li, X., Zang, C., Liversedge, S. P., \& Pollatsek, A. (2015). The role of words in Chinese reading. In A. Pollatsek \& R. Treiman (Eds.), The Oxford Handbook of Reading (pp. 232-244). Oxford University Press: Oxford.

Luke, S. G., \& Christianson, K. (2012). Semantic predictability eliminates the transposed-letter effect. Memory \& Cognition, 40, 628641. doi:https://doi.org/10.3758/s13421-011-0170-4

Morey, R. D. \& Rouder, J. N. (2011). Bayes factor approaches for testing interval null hypotheses. Psychological Methods, 16, 406-419. https://doi.org/10.1037/a0024377

Morey, R. D., Rouder, J. N., \& Jamil, T. (2015). Package 'BayesFactor'. Retrieved from https://cran.r-project.org/web/packages/ BayesFactor/

Norris D. (2013). Models of visual word recognition. Trends in Cognitive Sciences, 17, 517-524. doi:https://doi.org/10.1016/j.tics.2013.08. 003 
Perea, M., \& Lupker, S. J. (2003). Transposed-letter confusability effects in masked form priming. In Kinoshita S.and Lupker S. J. (Eds.), Masked priming: The state of the art (pp. 97-120). New York: Psychology Press.

Phillips, N. (2017). Package 'Yarrr'. Retrieved from https://CRAN.Rproject.org/package=yarrr

Rayner, K. (1975). Parafoveal identification during a fixation in reading. Acta Psychologica, 39, 271-281. doi:https://doi.org/10.1016/00016918(75)90011-6

Rayner, K., \& Well, A. D. (1996). Effects of contextual constraint on eye movements in reading: A further examination. Psychonomic Bulletin \& Review, 3, 504-509.

Rayner, K., Li, X., Juhasz, B. J., \& Yan, G. (2005). The effect of word predictability on the eye movements of Chinese readers. Psychonomic Bulletin \& Review, 12, 1089-1093. doi: https://doi. org/10.3758/BF03206448

Rayner, K., White, S. J., Johnson, R. L., \& Liversedge, S. P. (2006). Raeding wrods with jubmled lettres: There is a cost. Psychological Science, 17, 192-193. https://doi.org/10.1111/j.1467-9280.2006. 01684.x

Staub, A. (2015). The effect of lexical predictability on eye movements in reading: Critical review and theoretical interpretation. Language and Linguistics Compass, 9, 311-327. https://doi.org/10.1111/lnc3. 12151

Taft, M., Zhu, X., \& Peng, D. (1999). Positional specificity of radicals in Chinese character recognition. Journal of Memory and Language, 40, 498-519. https://doi.org/10.1006/jmla.1998.2625

Taylor, H. R. (1978). Applying new design principles to the construction of an illiterate E chart. American Journal of Optometry and Physiological Optics, 55, 348-351. doi:https://doi.org/10.1097/ 00006324-197805000-00008
R Development Core Team. (2016). R: A language and environment for statistical computing. R Foundation for Statistical Computing, Vienna, Austria. Retrieved from https://www.R-project.org/

Vandekerckhove, J., Matzke, D., \& Wagenmakers, E. J. (2015). Model comparison and the principle of parsimony. Oxford handbook of computational and mathematical psychology, 300-319.

Venables, W.N., Ripley, B.D. (2002). Modern Applied Statistics with S, Fourth edition. Springer, New York. ISBN 0-387-95457-0, http:// www.stats.ox.ac.uk/pub/MASS4.

White, S., Johnson, R. L., Liversedge, S., \& Rayner, K. (2008). Eye movements when reading transposed text: The importance of word beginning letters. Journal of Experimental Psychology. Human Perception and Performance, 34, 1261-1276. https://doi.org/10. 1037/0096-1523.34.5.1261

Yang, J. (2013). Preview effects of plausibility and character order in reading Chinese transposed words: Evidence from eye movements. Journal of Research in Reading, 36, S18-S34. doi: https://doi.org/ 10.1111/j.1467-9817.2013.01553.x

Zhang, M., Liversedge, S. P., Bai, X., Yan, G., \& Zang, C. (2019). The influence of foveal lexical processing load on parafoveal preview and saccadic targeting during Chinese reading. Journal of Experimental Psychology: Human Perception and Performance, 45, 812-825. https://doi.org/10.1037/xhp0000644

Zhao, S., Li, L., Chang, M., Xu, T., Zhang, K., Wang, J., \& Paterson, K.B. (2019). Older adults make greater use of word predictability in Chinese reading. Psychology and Aging, 34, 780-790. https://doi. org/10.1037/pag0000382

Publisher's note Springer Nature remains neutral with regard to jurisdictional claims in published maps and institutional affiliations. 